\title{
Shell theorem for spontaneous emission
}

\author{
Kristensen, Philip Trøst; Mortensen, Jakob Egeberg; Lodahl, Peter; Stobbe, Søren
}

Published in:

Physical Review B Condensed Matter

Link to article, DOI:

10.1103/PhysRevB.88.205308

Publication date:

2013

Document Version

Publisher's PDF, also known as Version of record

Link back to DTU Orbit

Citation (APA):

Kristensen, P. T., Mortensen, J. E., Lodahl, P., \& Stobbe, S. (2013). Shell theorem for spontaneous emission. Physical Review B Condensed Matter, 88(20), [205308]. https://doi.org/10.1103/PhysRevB.88.205308

\section{General rights}

Copyright and moral rights for the publications made accessible in the public portal are retained by the authors and/or other copyright owners and it is a condition of accessing publications that users recognise and abide by the legal requirements associated with these rights.

- Users may download and print one copy of any publication from the public portal for the purpose of private study or research.

- You may not further distribute the material or use it for any profit-making activity or commercial gain

- You may freely distribute the URL identifying the publication in the public portal

If you believe that this document breaches copyright please contact us providing details, and we will remove access to the work immediately and investigate your claim 


\title{
Shell theorem for spontaneous emission
}

\author{
Philip Trøst Kristensen, ${ }^{1}$ Jakob Egeberg Mortensen, ${ }^{1}$ Peter Lodahl, ${ }^{2}$ and Søren Stobbe ${ }^{2}$ \\ ${ }^{1}$ DTU Fotonik, Technical University of Denmark, Ørsteds Plads 343, DK-2800 Kgs. Lyngby, Denmark \\ ${ }^{2}$ Niels Bohr Institute, University of Copenhagen, Blegdamsvej 17, DK-2100 Copenhagen, Denmark
}

(Received 29 January 2013; published 20 November 2013)

\begin{abstract}
We investigate spontaneous emission from excitons beyond the point source dipole approximation and show how the symmetry of the exciton wave function plays a crucial role. We find that for spherically symmetric wave functions, the Purcell effect is independent of the wave function size and therefore is given exactly by the dipole approximation theory. This surprising result is a spontaneous emission counterpart to the shell theorems of classical mechanics and electrostatics and provides insights into the physics of mesoscopic emitters as well as great simplifications in practical calculations.
\end{abstract}

DOI: 10.1103/PhysRevB.88.205308

PACS number(s): 42.50.-p, 42.70.Qs, 78.20.Bh, 78.67.Hc

The Purcell effect is the relative change in the spontaneous emission decay rate of an excited emitter due to the photonic environment. While originally derived for optical microcavities, ${ }^{1}$ the effect has been observed in a wide range of material systems including planar interfaces, ${ }^{2}$ photonic crystals (PCs), ${ }^{3}$ and near metal nanoparticles. ${ }^{4} \mathrm{~A}$ recent application has been to employ a controlled Purcell effect for extracting fundamental properties of semiconductor quantum dots (QDs), such as the oscillator strength and the internal quantum efficiency. ${ }^{5,6}$ By increasing the spontaneous emission rate, the Purcell effect can be utilized for overcoming the decoherence and dissipation processes inherent to QDs. For this reason, the Purcell effect has important applications in the generation of coherent photons from $\mathrm{QDs}^{7}$ and in solid-state optical quantum processing in general, ${ }^{8,9}$ and the Purcell effect remains a main driving force for nanophotonics research. Very large Purcell effects have been predicted for dipole emitters near nanoscale metal structures due to extreme field gradients, but recent studies have shown that nonlocal effects in the material response play a crucial role and that local theories can overestimate the Purcell effect by orders of magnitude. ${ }^{10}$ These results might suggest that nonlocal effects inside the emitter can smear out or average local variations in the photonic environment, e.g., near metallic nanostructures. Nevertheless, we prove a surprising theorem showing that for spherical emitters the nonlocal optical response inside the emitter cannot change the Purcell effect, and we illustrate that this result is remarkably robust against deviations from spherical symmetry. Interestingly, the theorem takes the form of a shell theorem for spontaneous emission.

Theoretically, the description of spontaneous emission from QDs is often performed using a framework originally derived for atoms and ions. It relies on the celebrated dipole approximation (DA), which greatly simplifies the theoretical description of light-matter interaction in cases where the wavelength is large compared to the extent of the emitter. For such point sources, the spontaneous emission decay rate factorizes as $\Gamma_{\mathrm{DA}}\left(\mathbf{r}_{0}\right)=\alpha_{\mathrm{QD}} \rho\left(\mathbf{r}_{0}\right)$, where $\alpha_{\mathrm{QD}}$ is intrinsic to the QD and $\rho\left(\mathbf{r}_{0}\right)$ is the local density of states (LDOS $)^{11,12}$ describing the photonic response from the environment at the QD position $\mathbf{r}_{0}$. The DA is a local response theory which has proven to be an excellent description for atoms and ions. ${ }^{13}$ For QDs, however, the DA is not valid a priori because of the extended size of QDs as compared to atoms and also because of the potentially rapid spatial variations of the fields in optical micro- and nanostructures. Indeed, the DA was recently found to be inadequate for describing measurements of spontaneous emission from QDs close to a silver mirror, ${ }^{14}$ and the symmetry of the exciton wave function was identified as a key property. Nevertheless, the impact of the wave function symmetry on the Purcell effect has not been investigated theoretically. Beyond the DA, the simple factorization of the spontaneous emission decay rate is no longer valid. In general, spontaneous emission is determined by a complex intertwining of light- and matter-degrees-of-freedom, which may be interpreted as the coherent back action of the radiated field from the extended emitter upon itself, as illustrated in Fig. 1(a). However, in this paper we show a surprising relation between the calculations within and beyond the DA: For spherically symmetric exciton wave functions, the Purcell effect is independent of the wave function size. Therefore, the Purcell effect is determined only by the photonic response at the emitter center; cf. Fig. 1(b). The result holds also for shell-type QDs, ${ }^{15,16}$ in which the exciton wave function $\chi_{\odot}(r)$ is a spherically symmetric shell around the core, as illustrated in Fig. 1(c). It thus bears a resemblance to the shell theorems
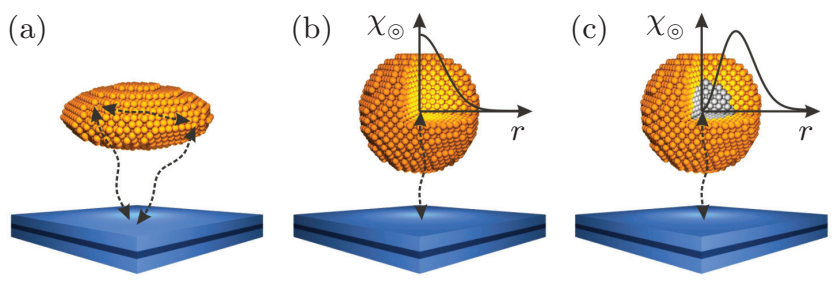

FIG. 1. (Color online) A shell theorem for spontaneous emission. (a) An extended emitter in inhomogeneous surroundings (exemplified by a QD above a dielectric mirror) coherently excites and scatters light (indicated by dashed lines) throughout the finite volume of the emitter itself. This may lead to nontrivial interference effects which significantly complicates Purcell effect calculations. (b) We derive a surprising theorem stating that for spherically symmetric exciton wave functions $\chi_{\odot}(r)$, the Purcell effect is determined only by the photonic response in the center of the emitter. (c) This nontrivial result is valid also for shell-type emitters in which the exciton wave function vanishes in the center. 
of classical mechanics ${ }^{17}$ and electrostatics, ${ }^{18}$ which state that the gravitational (electrostatic) force due to a spherically symmetric mass (charge) distribution is as if all the mass (charge) were concentrated in the center. Like the classical shell theorems, the relation dramatically simplifies practical calculations, because it effectively factorizes the decay rate as in the DA. For these reasons we regard it as a shell theorem for spontaneous emission.

Previous theoretical work on light-matter interaction beyond the DA has studied spontaneous emission from quantum dots and discs in bulk materials, ${ }^{19-21}$ near an interface ${ }^{22}$ or in the center of a spherical cavity, ${ }^{23}$ and the consequences of the DA breakdown on nonlinear effects was studied in Ref. 24. In this paper we study the impact of the exciton wave function symmetry on the Purcell effect using a rigorous yet intuitive Green tensor formalism ${ }^{25}$ for spatially extended exciton wave functions in QDs of different sizes and shapes and in two different material systems of current experimental interest: a PC cavity and a metal-dielectric interface. In light of these example calculations, we finally prove the shell theorem for spontaneous emission which is valid for general environments with arbitrary material distributions.

We consider spontaneous emission of light with angular frequency $\omega$ from excitons described by the wave function $\chi\left(\mathbf{r}_{\mathrm{e}}, \mathbf{r}_{\mathrm{h}}\right)$, where $\mathbf{r}_{\mathrm{e}}$ and $\mathbf{r}_{\mathrm{h}}$ denote the positions of the electron and hole, respectively. The wave functions $\chi\left(\mathbf{r}_{\mathrm{e}}, \mathbf{r}_{\mathrm{h}}\right)$ are completely different in the weak and the strong confinement regimes, ${ }^{25}$ but our analysis below is valid in both cases. Beyond the DA, the decay rate for spatially extended emitters can be written in the form ${ }^{22,25}$

$$
\Gamma(\chi)=\alpha_{\mathrm{NL}} \rho_{\mathrm{NL}}(\chi),
$$

in which $\alpha_{\mathrm{NL}}$ is determined by the crystal structure of the underlying QD material and includes the magnitude of the momentum Bloch matrix element. ${ }^{25}$ The nonlocal interaction function $\rho_{\mathrm{NL}}(\chi)$ is given as

$$
\rho_{\mathrm{NL}}(\chi)=\frac{2 \omega}{\pi c^{2}} \iint \chi(\mathbf{r}, \mathbf{r}) \mathbf{e}_{\mathbf{p}}^{\mathrm{T}} \operatorname{Im}\left\{\mathbf{G}\left(\mathbf{r}, \mathbf{r}^{\prime}, \omega\right)\right\} \mathbf{e}_{\mathbf{p}} \chi^{*}\left(\mathbf{r}^{\prime}, \mathbf{r}^{\prime}\right) d \mathbf{r} d \mathbf{r}^{\prime},
$$

where $c$ is the speed of light and $\mathbf{e}_{\mathbf{p}}$ is the orientation of the momentum Bloch matrix element. $\mathbf{G}\left(\mathbf{r}, \mathbf{r}^{\prime}, \omega\right)$ is the electric field Green tensor that we can interpret as the field at $\mathbf{r}$ from a point dipole at $\mathbf{r}^{\prime}$. The Green tensor is known explicitly for a number of simple geometries, such as free space and layered media, ${ }^{26-28}$ but in the general case it must be calculated numerically, for example, using time-domain formulations, ${ }^{29}$ integral equation techniques, ${ }^{30,31}$ or mode expansions. ${ }^{32,33}$ In Eq. (1) we have neglected nonradiative decay and broadening effects due to pure dephasing. These effects may be included in the formalism as an additional loss term and a stochastic Langevin noise term, ${ }^{34,35}$ respectively, but in order to focus specifically on the Purcell effect beyond the DA we have left them out. From Eq. (1) we define the Purcell factor as

$$
F_{\mathrm{P}}=\frac{\Gamma(\chi)}{\Gamma^{\mathrm{B}}(\chi)}=\frac{\rho_{\mathrm{NL}}(\chi)}{\rho_{\mathrm{NL}}^{\mathrm{B}}(\chi)},
$$

where $\Gamma^{\mathrm{B}}(\chi)$ and $\rho_{\mathrm{NL}}^{\mathrm{B}}(\chi)$ denote the decay rate and the nonlocal interaction function, respectively, in a homogeneous background medium described by the Green tensor $\mathbf{G}^{\mathrm{B}}\left(\mathbf{r}, \mathbf{r}^{\prime}, \omega\right)$. In the limit of small exciton wave functions, the nonlocal interaction function reduces to the LDOS, and the Purcell factor reduces to the relative LDOS, so that

$$
F_{\mathrm{P}}^{\mathrm{DA}}=\frac{\Gamma_{\mathrm{DA}}\left(\mathbf{r}_{0}\right)}{\Gamma_{\mathrm{DA}}^{\mathrm{B}}}=\frac{\rho\left(\mathbf{r}_{0}\right)}{\rho^{\mathrm{B}}},
$$

where "DA" refers to the rates as calculated in the DA, and $\rho^{\mathrm{B}}(\omega)=n_{\mathrm{B}} \omega^{2} / 3 \pi^{2} c^{3}$ is the LDOS in a homogeneous medium with refractive index $n_{\mathrm{B}}$. From Eqs. (3) and (4) we formulate the shell theorem for spontaneous emission in terms of the relative LDOS: For spherically symmetric exciton wave functions, indicated by "๑)" and centered on $\mathbf{r}_{0}$, the Purcell factor is exactly given by dipole theory:

$$
F_{\mathrm{P}}^{\odot}=\frac{\Gamma\left(\chi_{\odot}\right)}{\Gamma^{\mathrm{B}}\left(\chi_{\odot}\right)}=\frac{\Gamma_{\mathrm{DA}}\left(\mathbf{r}_{0}\right)}{\Gamma_{\mathrm{DA}}^{\mathrm{B}}}=F_{\mathrm{P}}^{\mathrm{DA}} \text {. }
$$

This is surprising because the DA can be vastly incorrect, ${ }^{25}$ and in general we find both $\Gamma\left(\chi_{\odot}\right) \neq \Gamma_{\mathrm{DA}}\left(\mathbf{r}_{0}\right)$ and $\Gamma^{\mathrm{B}}\left(\chi_{\odot}\right) \neq$ $\Gamma_{\mathrm{DA}}^{\mathrm{B}}$ but their ratios change in such a way as to keep the Purcell factor the same. From Eq. (5) it follows that $\Gamma\left(\chi_{\odot}\right)=$ $F_{\mathrm{P}}^{\mathrm{DA}} \Gamma^{\mathrm{B}}\left(\chi_{\odot}\right)$, so that the decay rate is simply the product of the Purcell factor in the DA and the background medium decay rate. This dramatically simplifies decay calculations beyond the DA, since $F_{\mathrm{P}}^{\mathrm{DA}}$ can be calculated by a variety of standard numerical techniques ${ }^{27-33,36,37}$ and depending on the exciton model, $\Gamma^{\mathrm{B}}\left(\chi_{\odot}\right)$ may be handled analytically. ${ }^{25}$

Before proceeding to a formal proof of Eq. (5), we first give a few example calculations. For an explicit exciton model we set $\mathbf{r}_{\mathrm{e} / \mathrm{h}}=\mathbf{r}=(x, y, z)$ and assume harmonic confinement potentials centered on $\mathbf{r}_{0}=\left(x_{0}, y_{0}, z_{0}\right)$, leading to Gaussian exciton wave functions,

$$
\chi(\mathbf{r}, \mathbf{r})=\chi_{0} e^{-\left|x-x_{0}\right|^{2} / \beta_{x}^{2}} e^{-\left|y-y_{0}\right|^{2} / \beta_{y}^{2}} e^{-\left|z-z_{0}\right|^{2} / \beta_{z}^{2}},
$$

where $\chi_{0}$ is a normalization constant. The size and geometry of the wave function is given by $\beta_{\alpha}, \alpha \in\{x, y, z\}$, and the model is a good approximation both in the weak and the strong confinement regimes, ${ }^{6,38}$ although the meaning of $\beta_{\alpha}$ differs in the two cases. ${ }^{25}$

As an initial, two-dimensional, example we set $\beta_{z}=0$ and consider disk-shaped QDs in an L3 cavity inside a finite-sized PC (lattice constant $a=220 \mathrm{~nm}$ and centered at $x=y=0$ ) of air holes with radius $r=0.26 a$ in a background medium with refractive index $n_{\mathrm{B}}=3.42$. The PC structure, which is shown in full in the inset in Fig. 2(b), supports the cavity mode s3 $^{33}$ shown in Fig. 2(a), for which the resonance wavelength in the material is $\lambda_{\mathrm{B}}=\lambda_{0} / n_{\mathrm{B}}=1.37 a \approx 300 \mathrm{~nm}$. Figure $2(\mathrm{~b})$ shows the Purcell factor in Eq. (3) at the cavity resonance and as a function of the QD position for two different orientations of elliptical QDs and two different radii of circular QDs. To prevent the exponential tails of large wave functions from reaching the air holes, we introduced an integration cutoff at the radius $R=2 \max \left\{\beta_{x}, \beta_{y}\right\}$. The estimated absolute errors in the calculated Purcell factors are less than 0.12 and stem primarily from a single-mode expansion of the Green tensor. ${ }^{32,33}$ Also, Fig. 2(b) shows the DA result in Eq. (4) which, in accordance with Eq. (5), clearly coincides with the Purcell factor calculations for the circular QDs even for $\beta_{x / y}>60 \mathrm{~nm}$. At the field node, in particular, it is striking that interaction with the field on either side of the node does not increase the Purcell 

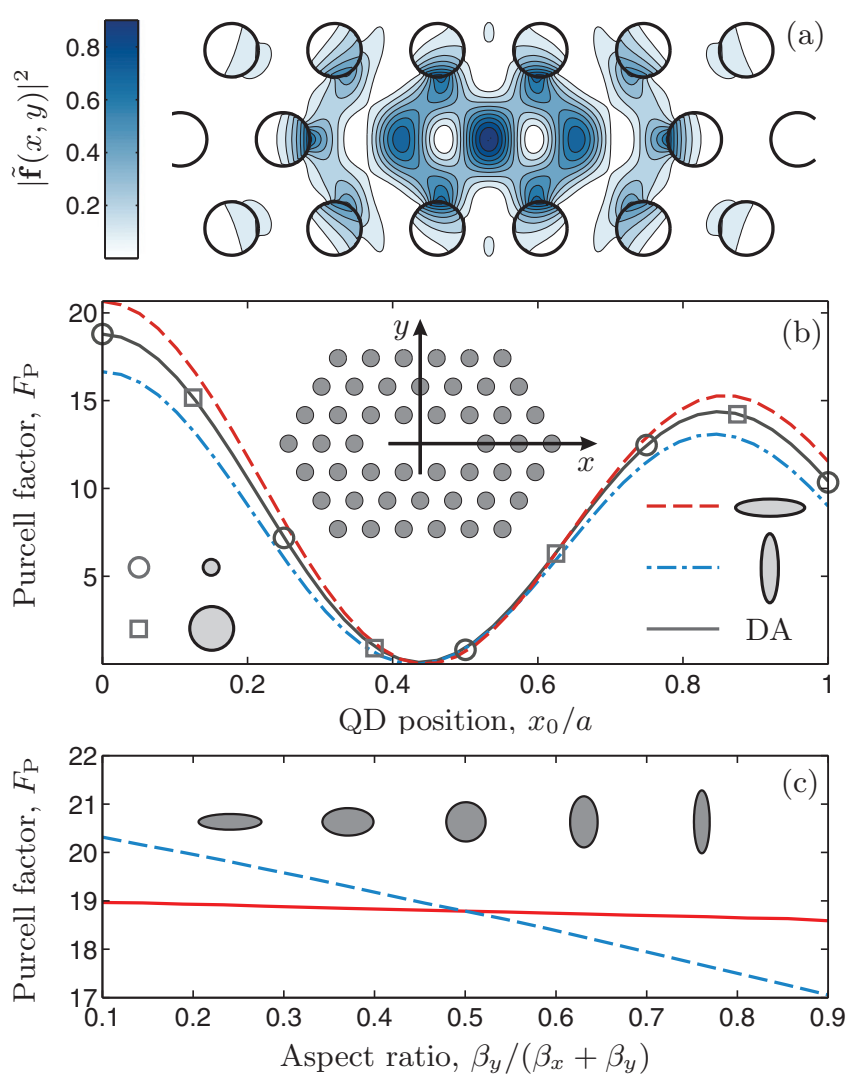

FIG. 2. (Color online) Purcell factors in a PC cavity. (a) Normalized mode profile $|\tilde{\mathbf{f}}(x, y)|^{2}$ of the PC cavity mode. (b) Purcell factor for $y$-polarized QDs as a function of QD position $x_{0}$ along the line $y_{0}=0$. For elliptically shaped QDs oriented along the $x$ axis ( $\beta_{x}=66 \mathrm{~nm}$ and $\beta_{y}=\beta_{x} / 10$, dashed red) or the $y$ axis $\left(\beta_{y}=66 \mathrm{~nm}\right.$ and $\beta_{x}=\beta_{y} / 10$, dashed-dotted blue) deviations from the DA (solid gray) are observable, but for circular QDs $\left(\beta_{x / y}=22 \mathrm{~nm}\right.$, circles; and $\beta_{x / y}=66 \mathrm{~nm}$, squares) the agreement with the DA is perfect, in accordance with Eq. (5). (c) Purcell factor at $x_{0}=y_{0}=0$ for $y$-polarized QDs of different shapes (see inset) and sizes; $\beta_{x}+\beta_{y}=$ $22 \mathrm{~nm}$ (solid red) and $\beta_{x}+\beta_{y}=66 \mathrm{~nm}$ (dashed blue).

factor above that of the DA, regardless of the QD size. In Fig. 2(c) we show the Purcell factor for QDs of different sizes and shapes at the field maximum $x_{0}=y_{0}=0$ in the cavity center. For $\beta_{x}+\beta_{y}=0.1 a=22 \mathrm{~nm}$ the DA is approximately valid and the QD shape has little influence, resulting in a nearly horizontal curve in the figure. For larger QDs with $\beta_{x}+\beta_{y}=$ $0.3 a=66 \mathrm{~nm}$ the different shapes do lead to changes in the Purcell factor, although the deviations from the DA are at most on the order of $10 \%$. The results suggest that the shell theorem in Eq. (5) is remarkably robust, even for very large and asymmetric QDs $\left(\beta_{x}>60 \mathrm{~nm}\right.$ and $\left.\beta_{x} / \beta_{y}=10\right)$. This has important implications for experiments on self-assembled QDs, where a circular symmetry is rarely present and electrons and holes experience different confinement potentials. ${ }^{39}$ Formally, for such geometries, one would have to calculate the Purcell factor using the general expression in Eq. (3). Nevertheless, our results suggest that the DA, when pertaining to the Purcell factor, is a good approximation for ground state excitons of self-assembled QDs in general PC structures.

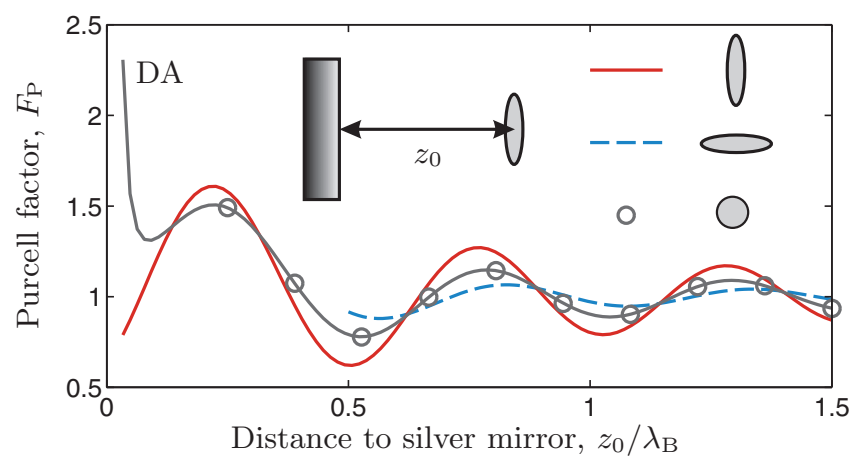

FIG. 3. (Color online) Purcell factor for $x$ - or $y$-polarized QDs of different shapes in GaAs as a function of distance $z_{0}$ to a silver mirror, as illustrated in the inset. For oblate ellipsoidal QDs $\left(\beta_{z}=5 \mathrm{~nm}\right.$ and $\beta_{x / y}=75 \mathrm{~nm}$, solid red) as well as for prolate ellipsoidal QDs ( $\beta_{x / y}=5 \mathrm{~nm}$ and $\beta_{z}=75 \mathrm{~nm}$, dashed blue) deviations from the DA result (solid gray) are evident, but for spherical dots $\left(\beta_{x / y / z}=50 \mathrm{~nm}\right.$, circles) the agreement with the DA is perfect.

As a second, three-dimensional, example we calculate the Purcell factor for QDs at different distances to a silver mirror with refractive index $n_{\mathrm{Ag}}=0.23+7.1 i$. The background medium has refractive index $n_{\mathrm{B}}=3.42$ and the wavelength is $\lambda_{\mathrm{B}}=300 \mathrm{~nm}$. We consider spherical and ellipsoidal QDs and assume that their polarizations are parallel to the mirror plane. Because of the simple geometry, and assuming the exciton to be in the background medium only, Eq. (2) reduces significantly and can easily be evaluated to arbitrary precision. The result is shown in Fig. 3 along with the DA result. We note that for oblate ellipsoidal QDs $\left(\beta_{x / y}=75 \mathrm{~nm}\right.$ and $\beta_{z}=5 \mathrm{~nm}$ ), the variations in the Purcell factor are larger than for the DA due to additional constructive and destructive interference throughout the volume of the QD. Conversely, for prolate ellipsoidal QDs $\left(\beta_{x / y}=5 \mathrm{~nm}\right.$ and $\left.\beta_{z}=75 \mathrm{~nm}\right)$, the variations are less pronounced. For spherical QDs the Purcell factor coincides with the DA results in the center of the QD in accordance with Eq. (5). In particular, there is no spatial averaging of the LDOS across the volume of the QD, despite the large field gradients near the metal. We note that for the larger nonspherical QDs, the additional interference effects lead to a nondivergent behavior close to the silver interface. This is in stark contrast to the DA result, which follows the relative LDOS and thus diverges as a well known consequence of the complex refractive index. The results in Fig. 3 suggest a need for relatively large QDs in order for effects beyond the DA to be visible. It is illustrative to compare this to the experimental results in Ref. 14 where the DA breakdown was observed for relatively small self-assembled QDs with radii on the order of $10 \mathrm{~nm}$. These QDs are known to be strained and asymmetric in the growth direction, but this asymmetry is not included in the exciton model in Eq. (6). Instead of elaborating the exciton model, however, we focus on the shell theorem for spherically symmetric exciton wave functions and leave the modeling of strained QDs for future studies.

Motivated by the example calculations in Figs. 2 and 3 we finally prove Eq. (5) by showing that for spherically symmetric exciton wave functions, $\chi(\mathbf{r}, \mathbf{r})=\chi_{\odot}(r)$, where $\chi_{\odot}(r)$ is an arbitrary function of radius $r$, the Purcell factor 
in Eq. (3) is independent of the emitter size. To this end, we first note that the Green tensor in general can be written as the sum of a background term and a scattering term, $\mathbf{G}\left(\mathbf{r}, \mathbf{r}^{\prime}, \omega\right)=\mathbf{G}^{\mathrm{B}}\left(\mathbf{r}, \mathbf{r}^{\prime}, \omega\right)+\mathbf{G}^{\mathrm{S}}\left(\mathbf{r}, \mathbf{r}^{\prime}, \omega\right)$, and from Eq. (3) it follows that we need only consider the fraction

$$
\frac{I_{\alpha \alpha}^{\mathrm{S}}}{I_{\alpha \alpha}^{\mathrm{B}}}=\frac{\iint \chi_{\odot}(r) \operatorname{Im}\left\{G_{\alpha \alpha}^{\mathrm{S}}\left(\mathbf{r}, \mathbf{r}^{\prime}, \omega\right)\right\} \chi_{\odot}^{*}\left(r^{\prime}\right) d \mathbf{r} d \mathbf{r}^{\prime}}{\iint \chi_{\odot}(r) \operatorname{Im}\left\{G_{\alpha \alpha}^{\mathrm{B}}\left(\mathbf{r}, \mathbf{r}^{\prime}, \omega\right)\right\} \chi_{\odot}^{*}\left(r^{\prime}\right) d \mathbf{r} d \mathbf{r}^{\prime}},
$$

where $G_{\alpha \alpha}^{\mathrm{S} / \mathrm{B}}\left(\mathbf{r}, \mathbf{r}^{\prime}, \omega\right)=\mathbf{e}_{\alpha}^{\mathrm{T}} \mathbf{G}^{\mathrm{S} / \mathrm{B}}\left(\mathbf{r}, \mathbf{r}^{\prime}, \omega\right) \mathbf{e}_{\alpha}$, and show that this is independent of $\chi_{\odot}(r)$. The QD is assumed to be fully embedded in a homogeneous and simply connected domain with refractive index $n_{\mathrm{B}}$, but it may be surrounded by materials with arbitrary permittivity distributions. The scattering term in the Green tensor represents light that is emitted at $\mathbf{r}^{\prime}$ and scatters in the environment to arrive at $\mathbf{r}$. It does not include light that travels directly from $\mathbf{r}^{\prime}$ to $\mathbf{r}$ and hence it is bounded for all $\mathbf{r}$ and $\mathbf{r}^{\prime}$. For the background term we note that the real part diverges in the limit $\mathbf{r} \rightarrow \mathbf{r}^{\prime}$. The imaginary part, however, is bounded for all $\mathbf{r}$ and $\mathbf{r}^{\prime}$, and therefore the imaginary part of both terms can be expanded as

$$
\operatorname{Im}\left\{\mathbf{G}_{\alpha \alpha}^{\mathrm{S} / \mathrm{B}}\left(\mathbf{r}, \mathbf{r}^{\prime}, \omega\right)\right\}=\sum_{m, n, p, q} c_{m n p q}^{\mathrm{S} / \mathrm{B}} \psi_{m n}(\mathbf{r}) \psi_{p q}\left(\mathbf{r}^{\prime}\right),
$$

in which $\psi_{m n}(\mathbf{r})=j_{n}\left(k_{\mathrm{B}} r\right) Y_{n}^{m}(\mathbf{r})$, where $j_{n}(r)$ and $Y_{n}^{m}(\mathbf{r})$ are spherical Bessel functions of the first kind and spherical harmonics, respectively, and $k_{\mathrm{B}}=n_{\mathrm{B}} \omega / c$. With Eq. (8), the integrals in Eq. (7) may be easily evaluated by noting that the angular integrations vanish for all terms except $m=n=p=$ $q=0$ and thus

$$
I_{\alpha \alpha}^{\mathrm{S} / \mathrm{B}}=4 \pi c_{0000}^{\mathrm{S} / \mathrm{B}}\left|\int_{0}^{\infty} \chi_{\odot}(r) j_{0}\left(k_{\mathrm{B}} r\right) r^{2} d r\right|^{2} .
$$

It follows that Eq. (7) is independent of $\chi_{\odot}(r)$ as it reduces to $I_{\alpha \alpha}^{\mathrm{S}} / I_{\alpha \alpha}^{\mathrm{B}}=c_{0000}^{\mathrm{S}} / c_{0000}^{\mathrm{B}}$. Finally, we remark on the limitations of the shell theorem. In general, the refractive index of the QD may be different from that of the surrounding medium, leading to local field effects ${ }^{40}$ and a possibly nontrivial dependence of the radiative lifetime on the QD radius. ${ }^{19}$ In such cases it might seem reasonable to include additional scattering from the QD itself in the definition of $\Gamma^{\mathrm{B}}(\chi)$, resulting in a possibly radiusdependent correction to Eq. (7). In many cases of practical interest, however, the requirements of the shell theorem will indeed be fulfilled because of the small refractive index contrasts between the QD material and the surroundings. ${ }^{21}$

In conclusion, we have investigated Purcell enhancement of spontaneous emission beyond the DA and illustrated how the symmetry of the exciton wave function has a decisive impact on the light-matter interaction. As a main result we have shown that for spherically symmetric exciton wave functions, the combined light-matter interaction of the exciton and the scattered field coherently cancels in such a way that the DA is exact for calculations of the Purcell effect. This result is a spontaneous emission counterpart to the shell theorems of classical mechanics or electrostatics and greatly simplifies the analysis of light-matter interaction beyond the DA. In addition, the result shows that asymmetric exciton wave functions must be employed in order to modify the Purcell factor by effects that go beyond the DA, and it thus provides important guidelines for design and interpretation of experiments aiming at measurements of such effects.

This work was supported by the VKR Center of Excellence NATEC and the Danish Council for Independent Research (FTP 10-093651 and FTP 10-080853).
${ }^{1}$ E. M. Purcell, Phys. Rev. 69, 674 (1946).

${ }^{2}$ K. H. Drexhage, J. Lumin. 1-2, 693 (1970).

${ }^{3}$ P. Lodahl, A. F. van Driel, I. S. Nikolaev, A. Irman, K. Overgaag, D. Vanmaekelbergh, and W. L. Vos, Nature 430, 654 (2004).

${ }^{4}$ D. Ratchford, F. Shafiei, S. Kim, S. K. Gray, and X. Li, Nano Lett. 11, 1049 (2011).

${ }^{5}$ J. Johansen, S. Stobbe, I. S. Nikolaev, T. Lund-Hansen, P. T. Kristensen, J. M. Hvam, W. L. Vos, and P. Lodahl, Phys. Rev. B 77, 073303 (2008).

${ }^{6}$ S. Stobbe, T. W. Schlereth, S. Höfling, A. Forchel, J. M. Hvam, and P. Lodahl, Phys. Rev. B 82, 233302 (2010).

${ }^{7}$ C. Santori, D. Fattal, J. Vučković, G. S. Solomon, and Y. Yamamoto, Nature 419, 594 (2002).

${ }^{8}$ D. E. Chang, A. S. Sørensen, E. A. Demler, and M. D. Lukin, Nat. Phys. 3, 807 (2007).

${ }^{9}$ J. L. O'Brien, A. Furusawa, and J. Vuckovic, Nat. Photonics 3, 687 (2009).

${ }^{10}$ C. Ciracì, R. T. Hill, J. J. Mock, Y. Urzhumov, A. I. FernándezDomínguez, S. A. Maier, J. B. Pendry, A. Chilkoti, and D. R. Smith, Science 337, 1072 (2012).

${ }^{11}$ R. Sprik, B. A. van Tiggelen, and A. Lagendijk, Europhys. Lett. 35, 265 (1996).

${ }^{12}$ L. Novotny and B. Hecht, Principles of Nano-Optics (Cambridge University Press, Cambridge, 2006).
${ }^{13}$ M. O. Scully and M. S. Zubairy, Quantum Optics (Cambridge University Press, Cambridge, 2001).

${ }^{14}$ M. L. Andersen, S. Stobbe, A. S. Sørensen, and P. Lodahl, Nat. Phys. 7, 215 (2011).

${ }^{15}$ A. Eychmüller, A. Mews, and H. Weller, Chem. Phys. Lett. 208, 59 (1993).

${ }^{16}$ J. Schrier and L.-W. Wang, Phys. Rev. B 73, 245332 (2006).

${ }^{17}$ I. Newton, The Mathematical Principles of Natural Philosophy, translated by A. Motte (Benjamin Motte, London, 1729).

${ }^{18}$ R. P. Feynman, R. B. Leighton, and M. Sands, The Feynman Lectures on Physics (Basic Books, New York, 2011).

${ }^{19}$ S. V. Goupalov, Phys. Rev. B 68, 125311 (2003).

${ }^{20}$ M. Sugawara, Phys. Rev. B 51, 10743 (1995).

${ }^{21}$ A. Thränhardt, C. Ell, G. Khitrova, and H. M. Gibbs, Phys. Rev. B 65, 035327 (2002)

${ }^{22}$ K. J. Ahn and A. Knorr, Phys. Rev. B 68, 161307 (2003).

${ }^{23}$ M. Glazov, E. Ivchenko, A. Poddubny, and G. Khitrova, Phys. Solid State 53, 1753 (2011).

${ }^{24}$ H. Ishihara, J. Phys.: Condens. Matter 16, R247 (2004).

${ }^{25}$ S. Stobbe, P. T. Kristensen, J. E. Mortensen, J. M. Hvam, J. Mørk, and P. Lodahl, Phys. Rev. B 86, 085304 (2012).

${ }^{26}$ O. J. F. Martin and N. B. Piller, Phys. Rev. E 58, 3909 (1998).

${ }^{27}$ M. Paulus, P. Gay-Balmaz, and O. J. F. Martin, Phys. Rev. E 62, 5797 (2000). 
${ }^{28}$ C.-T. Tai, Dyadic Green Functions in Electromagnetic Theory (IEEE Press, Piscataway, NJ, 1994).

${ }^{29}$ P. Yao, V. S. C. Manga Rao, and S. Hughes, Laser. Photonics Rev. 4, 499 (2010).

${ }^{30}$ D. P. Fussell, R. C. McPhedran, and C. Martijn de Sterke, Phys. Rev. E 70, 066608 (2004).

${ }^{31}$ P. T. Kristensen, P. Lodahl, and J. Mørk, J. Phys. Soc. Am. B 27, 228 (2010).

${ }^{32}$ K. M. Lee, P. T. Leung, and K. M. Pang, J. Opt. Soc. Am. B 16, 1409 (1999).

${ }^{33}$ P. T. Kristensen, C. V. Vlack, and S. Hughes, Opt. Lett. 37, 1649 (2012).
${ }^{34}$ G. Cui and M. G. Raymer, Phys. Rev. A 73, 053807 (2006).

${ }^{35}$ A. Naesby, T. Suhr, P. T. Kristensen, and J. Mørk, Phys. Rev. A 78, 045802 (2008).

${ }^{36}$ K. Busch and S. John, Phys. Rev. E 58, 3896 (1998).

${ }^{37}$ R. Wang, X.-H. Wang, B.-Y. Gu, and G.-Z. Yang, Phys. Rev. B 67, 155114 (2003).

${ }^{38}$ W. Que, Phys. Rev. B 45, 11036 (1992).

${ }^{39}$ P. W. Fry, I. E. Itskevich, D. J. Mowbray, M. S. Skolnick, J. J. Finley, J. A. Barker, E. P. O’Reilly, L. R. Wilson, I. A. Larkin, P. A. Maksym, M. Hopkinson, M. Al-Khafaji, J. P. R. David, A. G. Cullis, G. Hill, and J. C. Clark, Phys. Rev. Lett. 84, 733 (2000).

${ }^{40}$ K. Dolgaleva and R. W. Boyd, Adv. Opt. Photonics 4, 1 (2012). 